\title{
The Increasing Credibility of Sentinel Lymph Node Mapping in Endometrial Cancer
}

\author{
Nadeem R. Abu-Rustum, MD \\ Gynecology Service, Department of Surgery, Memorial Sloan-Kettering Cancer Center, New York, NY 10065
}

Since the pioneering study of Burke et al. from the MD Anderson Cancer Center, which was published in 1996, many investigators have explored the concept of sentinel lymph node (SLN) mapping in endometrial cancer. Two main issues have hindered progress in this area of research - the debate over the site of injection, with many investigators fixated on the view that a peritumoral injection, either hysteroscopic or fundal, is necessary to map disease, and the limited experience and small number of cases published over the 10 years following Burke's study (1996-2006). During the past 5 years, however, there has been a progressive, positive change in the way many gynecologic oncologists view the role of SLN mapping in endometrial cancer. This is partly due to the negative results of randomized trials on the therapeutic role of pelvic lymphadenectomy in unselected patients with endometrial cancer and the increasing awareness in the gynecologic oncology community about possible long-term side effects, such as lower extremity lymphedema from extensive lymphadenectomy in elderly patients. What adds to the pro-SLN argument is the lack of consensus on the extent of lymphadenectomy and the optimal anatomic templates (pelvic and aortic/renal) needed to stage these patients.

Most would agree that the identification of microscopic disease outside of the uterus can help to determine the need for adjuvant therapy; however, because the administration of adjuvant systemic therapy is increasingly based on uterine factors and not extrauterine findings, the role of staging to detect microscopic disease in peritoneal washings or random biopsies of normal-appearing omentum and

(C) Society of Surgical Oncology 2012

First Received: 14 August 2012;

Published Online: 6 October 2012

N. R. Abu-Rustum, MD

e-mail: abu-rusn@mskcc.org peritoneum may become more questionable. Also, most would agree that peritoneal washings or random biopsies of omentum or peritoneum that detect microscopic disease are not likely therapeutic but may alter the need for adjuvant therapy and prognosis. The same may be true for micrometastasis in normal-appearing lymph nodes. Lymph node removal for the purpose of staging is intended to detect microscopic disease that may guide the need for adjuvant therapy, which is increasingly relying on a combination of systemic chemotherapy and radiation.

If we believe that detecting nodal metastasis can guide the need for adjuvant therapy, then finding the nodes most likely to harbor disease would be paramount. SLN mapping is a form of image-guided surgery that can enhance our ability to find the more important nodes to test; in addition, having a pathologist examine these SLNs in an enhanced manner to detect micrometastasis and low-volume tumor spread, which may otherwise be missed with routine $H \& E$ examination, is extremely valuable. Both of these aspects are satisfied with the SLN algorithm approach to endometrial cancer staging. ${ }^{2}$ In 2011, Khoury-Collado et al. ${ }^{3}$ demonstrated that after a cervical injection, SLNs are three times more likely to harbor disease than non-SLNs; in addition, with enhanced pathology by serial sectioning and immunohistochemical ultrastaging, pathologists are able to detect an additional $3 \%$ of micrometastasis to SLNs, which may have been otherwise missed by routine H\&E.

The increasing validity of SLN mapping in apparent early-stage and low-to-intermediate risk endometrial cancer is further demonstrated by the data presented in this issue by Ballester and colleagues, with $15 \%$ of patients in this group having positive nodes and a significant proportion detected only by pathologic ultrastaging of the SLN. This is consistent with prior data in grade 1 endometrial cancers, in which $11 \%$ of these patients were found to have positive SLNs, ${ }^{4}$ and also consistent with recent data presented at Society of Gynecologic Oncology meetings, in 
which the incorporation of an SLN mapping protocol with pathologic ultrastaging allowed for the detection of $8 \%$ of positive regional nodes in a presumably low-risk group of patients who in some practices may not have undergone any nodal evaluation. ${ }^{5}$ This was further validated in a recent series of 401 cases in which the addition of pathologic ultrastaging allowed for the detection of nodal metastasis in a subset of patients in whom lymph node evaluation is not uncommonly altogether omitted. ${ }^{6,7}$ The data by Ballester and colleagues, as well as other emerging reports from around the world, support the incorporation of an SLN mapping algorithm in endometrial cancer staging, particularly for the presumed low-risk group. The distinctive findings in patients with grade 1 and grade 2 tumors argue strongly for the use of lymphatic mapping in this population in whom lymph node evaluation is not uniformly performed.

CONFLICTS OF INTEREST No conflicts of interest to disclose.

\section{REFERENCES}

1. Burke TW, Levenback C, Tornos C, Morris M, Wharton JT, Gershenson DM. Intra-abdominal lymphatic mapping to direct selective pelvic and paraaortic lymphadenectomy in women with high-risk endometrial cancer: results of a pilot study. Gynecol Oncol. 1996;62:169-73.

2. Barlin JN, Khoury-Collado F, Kim CH, et al. The importance of applying a sentinel lymph node mapping algorithm in endometrial cancer staging: beyond removal of blue nodes. Gynecol Oncol. 2012;125:531-5.

3. Khoury-Collado F, Murray MP, Hensley ML, et al. Sentinel lymph node mapping for endometrial cancer improves the detection of metastatic disease to regional lymph nodes. Gynecol Oncol. 2011; $122: 251-4$.

4. Abu-Rustum NR, Khoury-Collado F, Pandit-Taskar N, et al. Sentinel lymph node mapping for grade 1 endometrial cancer: is it the answer to the surgical staging dilemma? Gynecol Oncol. 2009; 113:163-9.

5. Khoury Collado F, Murray M, Hensley ML, et al. Sentinel lymph node mapping for grade $1 / 2$ endometrial cancer with superficial myoinvasion: less is more. Gynecol Oncol. 2011;120(Suppl 1):S94 (abstract).

6. Khoury Collado F, Alchyib O, Kim C, et al. Incidence of sentinel lymph node metastasis in endometrial carcinoma: correlation with tumor grade and myometrial invasion. Gynecol Oncol. 2012; 125(Suppl 1):S157 (abstract).

7. Kim C, Khoury-Collado F, Barlin JN, et al. Nanometastases of endometrial carcinoma to sentinel lymph nodes: the contribution of pathologic ultrastaging. Gynecol Oncol. 2012;125(Suppl 1): S154 (abstract). 